\title{
África: Cuerpos colonizados, cuerpos como identidades
}

\section{Africa: Colonized Bodies, Bodies as Identities}

\author{
Josep Martí \\ Institució Milà i Fontanals. CSIC. Barcelona
}

\section{RESUMEN}

Dada la gran importancia que toda sociedad otorga al cuerpo, tanto a su conceptualización como a los criterios para su presentación social, no es de extrañar que el cuerpo ocupe un lugar central en los procesos de colonización y las políticas coloniales. En este artículo se analiza la relación que podemos establecer entre colonialidad y presentación social del cuerpo, sirviéndonos para ello de los nuevos enfoques de los estudios postcoloniales, así como de la teoría antropológica y sociológica sobre el cuerpo en general. A partir de casos concretos de la colonización europea en África, el artículo se centra especialmente en las prácticas relativas al uso de indumentaria, así como a las modificaciones corporales. Si el establecimiento de identidades es crucial para construir un orden social jerarquizado, estos aspectos concretos de la presentación social del cuerpo han tenido un papel relevante en los procesos de subalternización, siendo la misma noción de salvaje uno de los ejemplos más claros para ello.

Palabras clave: Cuerpo, Colonización, África, Indumentaria, Modificaciones corporales

\section{SUMMARY}

Given the great importance that all society attach to the body, regarding its conceptualization as well as the criteria for its social presentation, it is not surprising at all that the body occupies a central place in the processes of colonization and colonial policies. This article, based on the new approaches of postcolonial studies, as well as on anthropological and sociological theory on the body in general, discusses the relationship we can establish between coloniality and the social presentation of the body. Drawing on concrete cases of European colonization in Africa, the article focuses on practices related to the use of clothing as well as body modification. If the establishment of identities is crucial for building a hierarchical social order, these specific aspects of the social presentation of the body have had an important role in subalternization processes, the very notion of savage being one of the clearest examples.

Key words: Body, Colonization, Africa, Clothing, Body Modification 


\section{INTRODUCCIÓN ${ }^{1}$}

Al hablar de colonización y políticas coloniales son muchos los aspectos que hay que tener en cuenta y entre estos, uno de los que más conciernen al antropólogo es sin duda alguna el conjunto de procesos de asimilación cultural, un elemento imprescindible para situar dentro del sistema social aquello que se pretende controlar. En seguida pensamos, pues, en parcelas tan importantes para la vida social como son la lengua, la religión, la organización política (por ejemplo y para el caso africano la denominada destribalización), etc. Hay no obstante otro aspecto que, aunque conocido, merece que se le preste todavía una mayor atención: la colonización de los cuerpos.

Hay diferentes maneras de colonizar los cuerpos. Nuestra historia nos enseña que una de las que ha tenido más relevancia es su minusvaloración mediante las teorías raciales que han surgido en un contexto de colonialidad. De esta manera se construían relaciones jerárquicas recurriendo al diferente color de la piel, las características del cabello o las medidas craneanas. Pero además de estos aspectos que tanta relevancia han tenido para el racismo, lo que podemos entender como colonización corporal se manifiesta también a través de los procesos aculturadores relativos a la presentación social del cuerpo.

El cuerpo ocupa un papel central en todo lo que tiene que ver con la colonialidad, es decir con todo aquel conjunto de patrones de poder que mediante la naturalización de jerarquías como las raciales o de género posibilitan la "re-producción de relaciones de dominación territoriales y epistémicas, que no sólo garantizan la explotación por el capital de unos seres humanos por otros, a escala mundial, sino que también subalternizan y obliteran los conocimientos, experiencias y formas de vida de quienes son así dominados y explotados" (Restrepo 2007: 292). Si es cierto - tal como nos enseña la antropología- que el cuerpo es un vehículo para la imposición de fuerzas sociales, políticas y económicas a individuos y a grupos, y al mismo tiempo también vehículo de resistencia a estas fuerzas (Reischer

${ }^{1}$ Trabajo realizado dentro del marco de los proyectos de investigación de I+D+I La presentación social del cuerpo en el contexto de la globalización y la multiculturalidad (SEJ2007-60792) y Cuerpo y procesos de modernización en África. El caso de Guinea Ecuatorial (CSO2011-23718). Parte del contenido de este artículo fue presentado en las "I Jornades d'Àfrica i Amèrica" (Universitat de Barcelona, Barcelona, 6-7 de mayo de 2010). Agradezco a Ana Lúcia Sá, así como a los evaluadores anónimos de la revista las sugerencias y observaciones realizadas en su lectura previa del manuscrito. 
y Koo 2004: 308), está claro que toda la problemática del cuerpo puede tener mucho que ver con la colonialidad. Para el caso particular africano y en palabras de Hildi Hendrickson: "clothing and other treatments of the body surface are primary symbols in the performances through which modernity — and therefore history - have been conceived, constructed and challenged in Africa" (1996: 13).

El jurista Tomás López Medel, en un informe que realizó en el siglo XVI sobre sus observaciones entre las poblaciones de tierras americanas, escribió:

En su vestir y traje es la gente de menos artificio que hay en todo el mundo. [...] Unas hay que así como nascen desnudos, desnudos se crían; acaban sus vidas ellos y ellas sin ropa alguna que traigan en todo su cuerpo ni en cabeza, ni género alguno de calzado en los pies, ni otra cosa en todo su cuerpo, más de como naturaleza les echó en el mundo, excepto que las mujeres por su honestidad y para cubrir sus vergüenzas traen un breve y corto paño de algodón, porque entre ellos no se usa otro lienzo, ceñido por el cuerpo y colgando hacia abajo a manera de un delantal y mandil. Esto acontece en indios de tierras calientes, y no es tan raro que no se vea en muchas partes y provincias de aquel Nuevo Mundo (López Medel 1990: 308).

Descripciones como esta las encontramos con mucha frecuencia en textos que hablan de la vida de pueblos conquistados. Y no nos debe extrañar tampoco la observación que sigue al anterior párrafo mencionado:

Verdad es que ya con la presencia de los españoles está más moderada [la criticada desnudez]; y como los indios se van baptizando, se van también en esto reformando ellos y ellas (López Medel 1990: 308).

Vestir, o más bien dicho, hacer vestir a la población colonizada según los patrones culturales de los conquistadores se ha entendido siempre como "obra de civilización", algo fácil de entender, dado que todos los sistemas sociales se han esforzado por regular la presentación social del cuerpo. Sólo hace falta que nos fijemos en nuestra propia sociedad. Salta a la vista la uniformización en materia de indumentaria que presentamos -a pesar de marcas y diferentes modelos de nuestros atuendos. $\mathrm{Y}$ especialmente indicativas al respecto son las controversias que surgen en ocasiones cuando se rompen determinadas normas establecidas; controversias por ejemplo con los nudistas cuando se dejan ver fuera de los guetos que tienen estipulados (Martí 2010b), o controversias cuando en nuestro país una mujer se muestra en público con el cuerpo cubierto por el nikab o — como se ha producido en alguna ocasión - también cuando una adolescente musulmana pretende ir al instituto con el pañuelo en la cabeza.

Entre las diferentes dimensiones de análisis que se han establecido para 
el estudio del cuerpo desde una perspectiva antropológica hay dos que son especialmente relevantes para el tema que nos ocupa ahora: el cuerpo social y el cuerpo político (Scheper-Hughes y Lock 1987: 7 y ss.). El cuerpo social tiene que ver con los usos representacionales del cuerpo en su calidad de símbolo natural. Este nivel de análisis nos hace pensar en cómo el cuerpo y sus productos (leche, sangre, saliva, esperma...) opera como símbolo natural, como útil o manera de representar relaciones sociales de la más diversa índole, como, por ejemplo, género, parentesco, clase social, etnicidad, edad, así como nociones de cariz jerárquico que en determinadas situaciones puntuales se pueden también desprender de estas categorías. El cuerpo político, basándose en la dimensión simbólica del cuerpo, hace referencia a cómo el cuerpo humano se usa como herramienta o arma de domesticación y disciplinamiento, y de identificación, sometimiento y resistencia. El cuerpo puede ser instrumentalizado políticamente precisamente porque constituye un "punto de imputación por excelencia del campo simbólico" (Le Breton 2002: 32). Y esta manera de percibir el cuerpo y de instrumentalizarlo tiene obviamente repercusiones directas en cómo se produce la experiencia vivida del cuerpo individual.

La problemática de la presentación social del cuerpo gira en torno a cómo mostramos el cuerpo ante los otros. Si, tal como se avanzaba en la introducción de este dossier, entendemos por presentación social del cuerpo la manera consciente y voluntaria de disponer de él en vistas a su interacción social mediante todo aquello que se hace en él, en otras palabras, la imagen que performativamente queremos dar del cuerpo, resulta evidente que básicamente son tres los ámbitos diferenciados que podemos determinar y que aparecen como significantes para nuestra presentación corporal (Martí 2010a): El cuidado general del cuerpo, la indumentaria y las modificaciones corporales. Mediante la presentación social del cuerpo, el yo/ propia identidad se engarza con el medio social y, a través de la performatividad —en el sentido butleriano del término (Butler 1988) — propia de esta presentación, se produce interactivamente el yo social.

La gran importancia que tiene la presentación social del cuerpo en los grupos humanos subyace en el hecho de que no es tan solo expresión del sistema social, sino también un valioso instrumento potenciador de la percepción colectiva de la realidad del sistema. De ahí, por ejemplo, el sentido de los uniformes. Por esta razón, los cambios sociales conllevarán cambios en la presentación social del cuerpo. Ello es así en cualquier sociedad, y, por ejemplo, muy fácil de observar en la pérdida de muchas tradiciones propias del África negra, relativas a la presentación social del cuerpo a partir especialmente de los procesos de colonización y los posteriores flujos de la globalización. 
La presentación social del cuerpo constituye un elemento de suma importancia dentro del sistema social y obviamente no resulta difícil ponerla en relación con otras facetas, más allá de la estricta dimensión identitaria, como el ethos general del sistema, la organización social o los medios de producción. Para el caso concreto que nos ocupa, la colonización en África, solo hace falta pensar, por ejemplo, en las relaciones que se han establecido entre indumentaria y moral, religión, estratificación social, etc. Las medidas relativas a la presentación social del cuerpo promovidas por los colonizadores son, no solo expresión desde el punto de vista ideacional de un sistema, sino que también tienen sus repercusiones económicas. El explorador inglés Henry Morton Stanley, en un discurso que hizo a la cámara de comercio de Manchester no se olvidó de mencionar los pingües beneficios que para los telares de aquella ciudad significaba el arduo trabajo que realizaban los misioneros en África para vestir a los que hasta entonces no tenían necesidad alguna de ropa: "There are 40 million naked people on the other side of the rapids, and the cottonspinners of Manchester are waiting to clothe them" (Manchester Chamber of Commerce 1884: 26).

Una asimilación, pues, no tan solo en relación a las normas morales, sino también a las necesidades de mercado. En el caso de la indumentaria, sus implicaciones económicas están bien claras, pero en ocasiones incluso podemos detectar implicaciones de este tipo en otras facetas relativas a la presentación social del cuerpo. Así, por ejemplo, entre los kikuyu, las mutilaciones genitales tanto masculinas como femeninas tienen una gran importancia como rituales de iniciación de los jóvenes y —según la cosmovisión tradicional- los hombres y las mujeres no son vistos socialmente como tales si no han pasado antes por estos rituales. Cuando estas prácticas, entendidas como ritos paganos por los misioneros, fueron prohibidas en los años 30 del siglo XX, los kikuyu mostraron una férrea oposición a esta prohibición pues, además de la importancia del ritual para los procesos de reproducción social, se entendió que estas directivas de las autoridades coloniales obedecían a intereses de los colonos para apropiarse de sus tierras. Si los jóvenes no devenían adultos — según el ethos local debían pasar antes por la circuncisión- los sacrificios destinados a los ancestros, identificados con la propiedad de la tierra no se podrían realizar. De esta manera, sin la protección de los espíritus de la tierra, el dominio familiar no existiría como tal y podría ser vendido a los colonos (Droz 2009: 124). 


\section{CUERPOS COLONIZADOS}

En un trabajo anterior hice ya alusión a tres diferentes parámetros de la lógica social que tienen una gran importancia para la incardinación de los individuos en las estructuras de cualquier sociedad: la identidad, el orden y jerarquización social, y la necesidad del intercambio (Martí 2010a). Se trata de tres criterios estrechamente relacionados y que son consubstanciales a la naturaleza humana como seres sociales que somos. Las identidades constituyen elementos imprescindibles para construir los encasillamientos sociales y estos determinan en muy buena medida los diferentes tipos de intercambio socialmente regulados entre los individuos: La lógica social nos pide saber quiénes somos dentro del colectivo, qué lugar ocupamos en las diferentes situaciones de jerarquía y orden social, y qué y con quién intercambiamos prestaciones en una situación determinada. Y todo esto lo expresamos también mediante la presentación social del cuerpo, precisamente por el alto contenido simbólico que se le otorga. De ahí que se pueda entender la "body surface as a principal site of social and political action" (Hendrickson 1996: 3).

La forma cómo se aplican estos tres parámetros en la visión del colonizador sobre el colonizado viene caracterizada por la existencia de determinados ejes semánticos basados en las dicotomías básicas nosotros-los otros, superioridad-inferioridad y civilizado-salvaje. Obviamente, estos tres ejes semánticos están estrechamente relacionados. En el ámbito de la colonización, la idea de superioridad del nosotros en relación a los otros se basa en toda una serie de narrativas que se sustentan, tanto en teorías pseudocientíficas de cariz racial a partir de la craniometría, frenología, fisiognomía o psicometría, por ejemplo — todas ellas pretendiendo determinar rasgos de personalidad e inteligencia a partir de características físicas del cuerpo-, como en la idea de superioridad cultural que descansa en la dicotomía civilizado-salvaje, que se corresponde con la vieja distinción ratzeliana entre Kulturvölker y Naturvölker. El salvaje, entendido también como homo infantilis (Sánchez Molina 2002; véase también Nandy 1983: 11-18; Fanon 1973: 26), es, por ejemplo, aquel que se muestra desnudo en el ámbito público o que se modifica el cuerpo con deformaciones o mutilaciones que no coinciden con las que en Occidente se aceptan por tradición. A partir precisamente de estas dicotomías se adscriben unas identidades, se las enmarca en un esquema de orden y jerarquía social, y se establecen e imponen unos patrones de intercambio según los intereses y worldview de los administradores coloniales. Si ahora aplicamos este modelo a la problemática que estamos tratando - la presentación social del cuerpo- pode- 
mos llegar a comprender fácilmente la importancia que esta cuestión puede llegar a tener para el ejercicio de la colonialidad.

La dominación colonial —en el sentido más amplio del término— se caracteriza por la coexistencia de dos fuerzas aparentemente contradictorias pero bien lógicas dentro de la dinámica de dominación. Por una parte la asimilación, por la otra la diferenciación. La asimilación es necesaria para que los pueblos colonizados formen parte de un sistema y puedan serle útiles; nos movemos por tanto en el ámbito de la lógica social de la identidad y del intercambio. La diferenciación es necesaria para garantizar unas relaciones de jerarquía que son consubstanciales al pensamiento colonial. Esto tiene también que ver con las lógicas de la identidad y de la regulación del orden y jerarquías sociales. Y todo esto se produce según la dinámica de lo que se ha denominado la colonialidad del poder. Una asimilación que no se efectúa solo de manera explícitamente forzada, sino naturalizando el imaginario cultural europeo como forma única de relación con la naturaleza, con el mundo social y con la propia subjetividad; una asimilación que pretende cambiar radicalmente las estructuras cognitivas, afectivas y volitivas del dominado, convirtiéndolo en una nueva persona hecha a imagen y semejanza del dominador (Castro-Gómez 2005: 59).

Por lo que se refiere a los procesos de asimilación cultural en relación con la presentación social del cuerpo, para el caso africano, lo primero que nos viene a la mente es la cuestión de la desnudez. Sabemos que el pudor corporal se construye mediante mecanismos culturales y por tanto resulta obvio que se entienda de manera diferente según la sociedad y el espacio temporal en cuestión. Son muchas las poblaciones del planeta que antes del contacto con otras civilizaciones no sentían la necesidad de cubrir el cuerpo por razones de pudor. Por lo que se refiere al caso particular africano, en muchas de estas poblaciones el hábito de cubrir el cuerpo por pudor se asumió al entrar en contacto directo con el cristianismo o con el islam, y muy especialmente en situaciones de colonialismo. Obviamente, la adopción de estos nuevos valores se explica por la desigual relación de poder — real y simbólico- entre colonizadores y colonizados, además del celo que administradores y misioneros mostraron para imponer los propios valores en cuanto a ideas morales y de decencia se refiere. Esta imposición resultó patética en aquellos casos en que se obligaba a los autóctonos a usar una indumentaria en absoluto apropiada para las condiciones climáticas y de vida, circunstancia que incluso conllevó verdaderos problemas de higiene y salud. Que la desnudez, o relativa desnudez, tenía en África tropical su lógica intrínseca era algo que los viajeros no ponían en duda: 
We wonder at them how they can go naked; but they wonder more at us, how we can endure to go packed up in a fardle of clouts sewed together. We are bound up in prison whilst they are free and at liberty. We are stifled up in our sweat and stink whilst the exhalations of their vapors offends them not. Nor doth the coldness of the air hurt their naked bodies more than it doth our naked faces. It was our evil custom that clothed us, and their innocency and freedom of nature that keeps them naked (Hamond 1640, citado en Wright 1943: 115-116).

A principios del siglo XIX, el explorador Richard Lemon Lander hablaba con una cierta envidia de la poca indumentaria que llevaban los bubi de la isla de Bioko, Guinea Ecuatorial, dado que por las razones climáticas imperantes, «todos desearíamos ir como ellos, fuera naturalmente de embadurnarnos el cuerpo con barro y aceite" (Lander y Lander 1844: 301 v.2, citado en Martin del Molino 1994: 83). Algunas décadas más tarde, no obstante, en la misma isla, el gobernador español de Santa Isabel - hoy Malabo- prohibió la entrada a la ciudad a los autóctonos que fueran desnudos (Beltrán y Rózpide, citado en Nerín 1998: 100).

En Occidente se relaciona el ir vestido con civilización. En realidad, sin embargo, difícilmente encontraríamos grupos humanos que se presenten completamente desnudos. O tal como afirmaban Esther y Jack Goody, "ir vestido no es lo mismo que llevar vestido" (Goody y Goody 1996: 68). A pesar de que no se cubra el cuerpo con ropa, hay otras maneras de $\mathrm{cu}$ brirlo o vestirlo como la escarificación, el tatuaje o la pintura, solo que obviamente estos no eran los criterios de los colonizadores occidentales. Al amparo del eje semántico civilizado-salvaje era necesario vestir a los que iban desnudos. Esto, como es bien sabido, se promovía mediante disposiciones de las autoridades coloniales, como la del ejemplo antes mencionado. Pero del combate contra la desnudez se encargaban de manera mucho más sistemática y voluntariosa los misioneros cristianos o bien las autoridades religiosas musulmanas en sus ámbitos de penetración. De hecho, la apropiación de indumentaria europea se consideraba un hecho crucial en la creación de una cultura cristiana local (Ruether 2002: 370). Bien sintomático es este texto extraído de la revista claretiana La Guinea Española:

En el verano de 1928 acompañadas por las Hermanas Misioneras, vinieron a Italia unas negritas de Somalia. En el Instituto de una ciudad italiana un grupo de Aspirantes y Benjaminas las obsequiaron con una velada. Una de las negritas dijo a una Benjamina, que estaba sentada con las faldas un poco levantadas: «Siéntate bien: de lo contrario nuestra Hermana te regañará". Aquella contestó amostazada: "Pero, vosotras en África no lleváis vestidos". Repuso la negrita amablemente: "Cuando éramos salvajes, no; pero ahora que somos cristianas, sí. Vamos vestidas y más cubiertas que vosotras" (Ruiaz 1944: 21).

"Cuando éramos salvajes" no llevábamos vestidos. Vestir, pues, no era 
tan solo sinónimo de asimilación al sistema, era también dotar de dignidad (o de una cierta dignidad) a los que se consideraba que no la tenían -recordemos que para Kant ir vestido equivalía a dignidad- y era también difundir una moral y creencias. Según la perspectiva etnocéntrica del eje semántico nosotros-los otros se trataba tout court de humanización: "La verdadera colonización [...] es ante todo humanización y cristianización" (Aguayo 1943: 260). En los primeros tiempos de la dominación colonial de la Ghana actual, el grado de indumentaria servía para comparar el nivel de civilización entre los colonizados, así como de índice para medir los avances hacia la civilización de las tribus desnudas y salvajes (Allman 2004: 147). Y de hecho, para la antropología social, la adopción de indumentaria europea junto con otros índices, tales como la educación, las ocupaciones laborales y los ingresos, han constituido unos de los principales indicadores para los procesos de aculturación y de cambio, y la formación de nuevos grupos de estatus en el contexto urbano africano (Magubane 1971: 419).

La desnudez, así como determinados tipos de modificación corporal, han sido aprovechados por las narrativas coloniales para construir la noción de "salvaje" o "primitivo" y por tanto para dotar a los colonizados de características culturales que, situando estos pueblos en estadios inferiores, dentro de una jerarquización evolutiva permitía justificar las desigualdades sociales y económicas entre colonizados y colonizadores (Heinz 1998: 427). Estas ideas las encontramos constantemente en publicaciones de la escena colonial, como es el caso, por ejemplo, de la revista La Guinea Española:

Las nuevas ideas, base de una educación muy diferente y el contacto con una cultura superior, suaviza el cauce de una vida salvaje: se destierra el tatuaje: lo suyo hubo de costar cuando de hecho se opuso el gobierno a la vigencia de una costumbre absurda por inhumana (Ruiaz 1944: 91).

En un artículo de la misma revista, el misionero claretiano Antonio Aymemí, que entendía que las escarificaciones faciales de los bubi en la isla de Bioko los hacía "horriblemente feos, desfigurados y repugnantes" (Aymemí 1942: 25), nos cuenta la historia de un bubi, considerado por los misioneros buen cristiano, que en 1905 vivía en Batete con tres hijos y que "ninguno de ellos había sufrido el bárbaro tatuaje bubi" ${ }^{2}$. Un cierto día se le presentó a Aymemí el mayor de aquellos muchachos "con la cara horriblemente desfigurada".

\footnotetext{
${ }^{2} \mathrm{El}$ autor se refiere a cortes realizados en la cara en forma de líneas paralelas y que podían ser bastante profundos. Se practicaba durante la infancia, tanto a hombres como a mujeres.
} 
Llamé a su padre y reprendile severamente por tan abominable conducta con su propio hijo. Contestome con calma el buen hombre diciendo de este hecho no soy responsable, ni tengo nada que ver con ello. Yo soy quien engendró al muchacho, pero la ley me niega los derechos que supone Vd. tengo sobre él, por la sencilla razón de [que] la madre es viuda propia de otro que la compró, la cual está unida a mí mediante el ribala rerijolé [aparejamiento sin matrimonio]. El muchacho es hijo natural mío pero legal de la familia del difunto marido de mi amiga, ella ha mandado tatuar la cara de mi hijo propiedad suya y yo no lo he podido impedir, ni oponerme a ello (Aymemí 1943: 47).

En el texto, al mismo tiempo que se refleja la profunda aversión que los colonizadores sentían por las escarificaciones faciales, se mencionan regulaciones propias de un sistema familiar que también se considerará incivilizado y que constituirá uno de los principales caballos de batalla de los misioneros en su tarea de civilizar al salvaje 3 . Muchos años antes, el pastor de la Iglesia Presbiteriana, Ibia Dy'Ikengue que realizó labores misioneras en Guinea Ecuatorial durante el siglo XIX, en un libro que se publicó en 1872, entendía como prácticas aberrantes los tatuajes, las escarificaciones y el ndjangui o costumbre de modificar el dentado dejando espacios libres entre los dientes. Y concretamente escribía: "Les aconsejamos a que abandonen estas prácticas. Mucha gente está malgastando sus cuerpos con estas prácticas absurdas" (Dy’lkengue 2004: 248-249).

Esta calificación de prácticas incivilizadas para tatuajes, escarificaciones u otros tipos de modificaciones corporales, es una de las razones más importantes para su progresiva desaparición a lo largo del siglo XX en África. Muy a menudo se decretaron prohibiciones explícitas por parte de las autoridades coloniales, como fue el caso de las escarificaciones en la isla de Bioko, pero sobre todo desaparecieron por el descrédito que las narrativas de los colonizadores les otorgaban, como sucedió —en el mismo país de Guinea Ecuatorial- con los tatuajes que los fang se practicaban en múltiples partes del cuerpo ${ }^{4}$.

La fuerza con que se implantaron y asimilaron las narrativas coloniales en las que se relacionaban determinados aspectos de la presentación social del cuerpo con primitivismo, hace que en África estas narrativas continúen plenamente vigentes después de la independencia de los países coloniza-

${ }^{3}$ De hecho no nos debe extrañar la gran importancia que la revista La Guinea Española, publicada con este nombre por los claretianos desde 1903 hasta 1968, otorga a la familia. Son numerosos los artículos centrados en la idea de familia, tanto los que ensalzan el modelo occidental como los que aportan datos etnográficos sobre esta institución entre las poblaciones autóctonas de Guinea Ecuatorial y que no hacen sino subrayar su pretendido salvajismo.

${ }^{4}$ Al respecto véase Sabater y Sabater (1992). 
dos, precisamente porque son narrativas que no se presentaban únicamente como propias de una potencia colonial en concreto, sino como exponentes de la civilización en abstracto, como elementos de modernidad canalizados por los poderosos procesos de globalización. Esto es especialmente cierto en la identificación de la oposición cuerpo desnudo-cuerpo vestido con la de salvaje-civilizado. Así, por ejemplo, en Tanzania a finales de los años 60, el gobierno postcolonial inició una campaña por razones de modernidad, denominada Operation Dress-Up, para que los maasai abandonasen su modo de vestir tradicional; un modo de vestir que no cubría de manera apropiada su desnudez. Se les amenazaba con el arresto y con otras medidas, como negar a quien fuese vestido de aquella manera la entrada a las escuelas, centros de asistencia médica, bares, restaurantes o transporte público (Schneider 2006: 105-106). Aproximadamente en la misma época, en Ghana, un año después de lograda la independencia, se iniciaron campañas para erradicar la desnudez en las zonas septentrionales del país que duraron varios años (Goody y Goody 1996: 82; Allman 2004: 144 y ss.), mientras que en el estado vecino de Costa de Marfil la desnudez estaba ya oficialmente prohibida (Allman 2004: 158). Incluso el reportero polaco Kapuscinski, dentro de su peculiar estilo, se hizo eco de estos intentos gubernamentales para desterrar la desnudez en África en su célebre obra Ébano. Al hablar de la costumbre de los karamajong de vivir en desnudez, cuenta que el despótico y cruel dictador de Uganda, Idi Amin, considerando que el hecho de ir desnudo ponía en ridículo a los africanos, amenazaba con fusilar en el acto a toda persona a la que se atrapase yendo desnuda en público (Kapuscinski 2004: 150) ${ }^{5}$. Los edictos contra la práctica de la desnudez —entendida en términos propios de la tradición occidentalcontinúan siendo una realidad en África ya bien entrado el siglo XXI (véase por ejemplo para Nigeria, Bakale-Yosuf 2011).

\section{CUERPOS COMO DEMARCADORES IDENTITARIOS}

Antes decíamos que la dinámica de la colonización implica, tanto la asimilación de los colonizados, como también la diferenciación identitaria para así continuar manteniendo una relación de jerarquía y posibilitar un régimen de intercambio basado de hecho en la explotación. La idea de que "el destino del negro era imitar al blanco", tal como por ejemplo se expresaba a finales de los años 1940 el gobernador general de la colonia

\footnotetext{
${ }^{5} \mathrm{Y}$ de hecho se produjeron matanzas por esta razón. Véase al respecto Mirzeler y Young (2000: 416).
} 
guineoecuatoriana (Bonelli 1949, citado en Medina-Doménech 2009: 89), es una idea bien establecida en la mentalidad colonial, aunque por otra parte también estaba claro que:

Civilizar al negro no debe ser en modo alguno convertirlo en europeo, sino organizado en una sociedad que tenga de nuestra civilización lo fundamental, o sea la religión y el amor a la Patria, pero que conserve de la sociedad primitiva cuanto no se oponga a esta civilización (Fontán 1943).

Son muchos los datos etnográficos que nos hablan del "entusiasmo" con que muy a menudo los colonizados adoptaron aspectos propios de la presentación social del cuerpo de los europeos, como por ejemplo la indumentaria; un "entusiasmo" por cierto que no puede desligarse del commodity racism (McClintock 1995: 207 y ss.). Pero si en un primer momento parece que esto tenía que cuadrar con la filosofía de los colonizadores, pronto despertó suspicacias por parte de los blancos cuando se pensaba que esta asimilación podía llegar a subvertir el orden y jerarquización social. Ruether, hablando de la actividad misionera alemana a finales del siglo XX en el Transvaal, reproduce comentarios críticos que los misioneros hacían sobre los usos de la indumentaria europea entre los autóctonos:

[...] with respect to clothing they are all interested in Europeans, and whenever somebody can manage or returns from the diamond fields, English Gentlemen can see that in his clothing he is no longer superior to a kaffir (Ruether 2002: 369).

Para la Kenia colonial encontramos parecidos ejemplos en los que se ridiculiza el uso por parte de africanos de indumentaria europea (Hay 2004: 68). En el caso concreto de los misioneros anglicanos, si bien se conmina a la población local a cubrir su desnudez, se tiende a desanimarla a que use el mismo tipo de indumentaria que el blanco (Hay 2004: 69).

Por eso no debe extrañar que en los momentos de la colonización se tuviera interés, no solo en vestir a los negros, sino muy a menudo también en controlar de una manera u otra su indumentaria. Así, por ejemplo, en muchas misiones se tomaron medidas para evitar que los feligreses convertidos usaran una indumentaria demasiado lujosa (Ruether 2002: 375). Phyllis Martin, hablando sobre indumentaria en Brazzaville, capital del África Ecuatorial francesa a finales del siglo XIX, nos menciona la insistencia de los blancos en recordar el papel subordinado de sus empleados locales mediante las convenciones del vestido: los criados tenían que vestir pantalones cortos y no se les permitía llevar calzado. Los tiradores, incluso los que se habían graduado en la escuela y estaban haciendo el servicio militar o los policías negros tenían que ir también descalzos (Martin 1994: 408).

Los occidentales llegaron a percibir el uso de indumentaria europea por 
parte de los africanos como una expresión directa de oposición a la supremacía blanca y a interpretarla en términos de inapropiadas aspiraciones sociales (Ruether 2002: 368). El deseo de lucir un traje civilizado por parte de africanos irritaba a muchos europeos en el Gabón de finales del siglo XIX; existía la impresión de que muchos africanos pretendían ocultar el color de su piel mediante el uso de vestidos europeos ${ }^{6}$. De hecho, generalmente y en un principio, los autóctonos no se oponían a la adopción de indumentaria europea, sino todo lo contrario, ya que se entendía esta apropiación como estrategia para reclamar igualdad (Ruether 2002: 366) o incluso para distanciarse de otros grupos étnicos o estratos sociales con los que se coexistía (Rich 2005: 195). Así, pues, tal como escribió James Ferguson, la presencia del "nativo civilizado" podía llegar a desestabilizar las identidades coloniales (2002: 552).

Y si esto sucede con la indumentaria, también hallamos ejemplos relativos al color de la piel, que resultan muy ilustrativos sobre la importancia

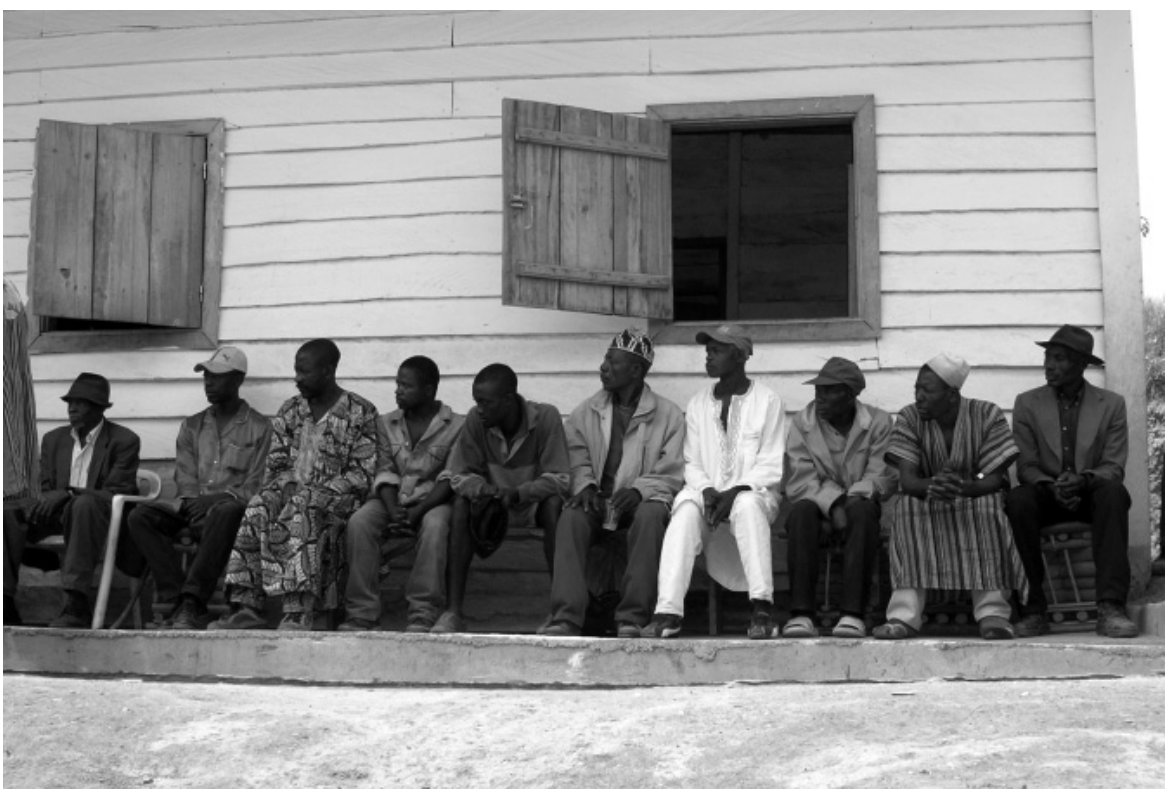

Figura 1.-En África, debido a las propias tradiciones, a la colonización y a la actual globalización, coexisten fácilmente múltiples estilos de indumentaria. Localidad rural de la región central del Camerún, 2008. Foto de J. Martí.

\footnotetext{
${ }^{6}$ "C'est pour faire oublier sa couleur détestée que le Noir, surtout le Noir 'conscient' [...] se surcharge de vêtements européens, de cols exagérés, de cravates éclatantes, de gants, de souliers jaunes ou vernis, de binocles, de dents aurifiées" (Briault 1943: 226).
} 
que se da al cuerpo en el marco de las identidades y su jerarquización social. En la primera década del siglo XX, se llevaron a cabo experimentos en EE. UU con radiación electromagnética y, en un primer momento, parecía que los rayos-X podían ser usados para blanquear la piel. Al hacerse esto público, se produjo una cierta alarma social canalizada por diversos medios de comunicación porque se temía que esta nueva tecnología no solo eliminaría la división visual entre blancos y negros, permitiendo hacer pasar a negros por blancos, sino que la blancura que podría llegar a obtener la piel negra podría superar incluso a la de muchos blancos (Peña 2006: 39).

Actualmente el blanqueamiento de piel constituye una práctica muy extendida en el África subsahariana, especialmente entre mujeres (Blay 2011: 22-23). En el mercado se ofrece una gran variedad de cosméticos que se usan con esta finalidad, en ocasiones con consecuencias claramente perjudiciales para el cuerpo, especialmente cuando estos productos contienen derivados del mercurio o hidroquinona ${ }^{7}$. En los últimos años son cada vez más los países africanos que intentan vetar la venta de estos productos en aquellos casos que son claramente dañinos para la salud (Blay 2011: 24), y debido a las ideas de tinte racista que subyacen en esta práctica, en Sudáfrica, ya en los años noventa del pasado siglo se prohibió la publicidad de cosméticos en la que se hiciera algún tipo de alusión al blanqueamiento de piel (Thomas 2011). Aunque se ha tachado el uso de estos cosméticos blanqueadores como algo "absurdo" (Miyanji de Souza 2008: 28) ${ }^{8}$, es obvio que se trata de una práctica perfectamente racional, al menos en el sentido de la limited rationality (Simon 1960). Las prácticas de modificación corporal de blanqueamiento de piel se pueden entender como recursos estratégicos para hacer frente al colorismo9 (Hunter 2007), la ideología que otorga un mayor valor a las personas con piel de tonalidad clara ${ }^{10}$.

${ }^{7}$ Actualmente existe una poderosa industria cosmética con esta finalidad. Aunque enfocada principalmente hacia el mercado asiático, también tiene al africano como uno de sus objetivos. Esta moderna industria se basa en procedimientos biotecnológicos para inhibir la producción de melanina en el cuerpo humano.

${ }^{8}$ La autora no tiene ningún reparo ni pudor en compartir la idea de que "Africa is considered a continent of mystery and intrigue with absurd concepts and beliefs" (Miyanji de Souza 2008: 27). Tal como hemos podido ver en otros ejemplos a lo largo de este mismo artículo, el uso del adjetivo "absurdo" es moneda corriente para referirse a prácticas relacionadas con las modificaciones corporales.

9 Término acuñado por Alice Walker (1983) y que de acuerdo con las palabras de Yaba Amgborale Blay constituye un sistema de percepciones jerárquicas de valor y tratamiento discriminatorio basado en la coloración de la piel (Blay 2011: 37).

${ }^{10}$ No obstante, hay que tener también en cuenta que, según algunos autores, la preferencia en África por colores de piel más claros no tiene que entenderse siempre 


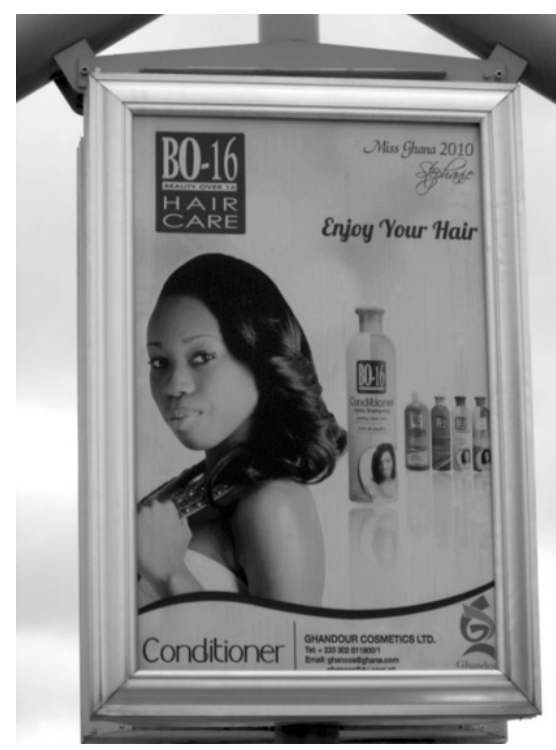

FIGURA 2.-En los anuncios publicitarios de cosméticos se presenta a menudo a la mujer con un tono de piel claro. Accra, Ghana 2011. Foto de J. Martí.

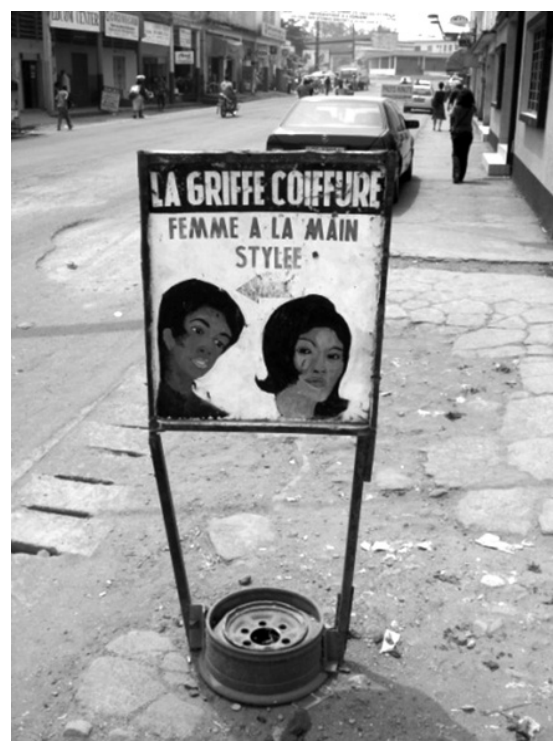

FIgURA 3.-Anuncio de una peluquería en el que destaca el color más claro de piel de la mujer respecto al hombre. Nkongsamba, Camerún 2008. Foto de J. Martí.

\section{LA CONTINUIDAD DE UN PARADIGMA}

La presentación social del cuerpo puede servir, pues, como recurso para reclamar igualdad, de la misma manera que también se puede recurrir a ella como estrategia para recordar las divisiones jerárquicas establecidas por el colonialismo. E incluso hoy podemos constatar la vigencia de esta última estrategia en el denominado primer mundo dado que nos viene bien recordar la inferioridad de aquellos otrora colonizados, con más razón ahora que los tenemos en casa y muchos sectores de la población los ven como potenciales competidores en la arena social. Así, dentro de la percepción del occidental, se consideran grotescos o estrafalarios determinados usos que la población africana hace de una indumentaria que seguimos reivindicando como occidental. En determinadas situaciones nos puede sorprender la ligereza con la que estos africanos combinan piezas de ropa de cariz formal con informal, criticamos soto voce el uso de vistosos trajes y corbatas

forzosamente dentro de la lógica del colonialismo occidental, sino que en ocasiones obedece a criterios locales sobre la apariencia corporal (Ardener 54: 71; Fortes 1945: 7). 
en situaciones en las que al europeo le pueden parecer inapropiadas ${ }^{11}$, y prácticas tan llamativas como las de los sapeurs del Congo ${ }^{12}$ nos pueden resultar difíciles de comprender, dado que si son pobres, estos vistosos pantalones, americanas y zapatos inspirados en la alta moda parisina nos parecen un ridículo despilfarro de recursos y una tergiversación del mensaje que adscribimos a este tipo de indumentaria en nuestro contexto social: "Marcas prestigiosas, clase y un gusto incontestables no dejan de provocar asombro cuando los dandis viven en condiciones muy precarias" (Mediavilla 2008: 51). La idea de fondo que no resulta difícil de captar en estas percepciones es que tienen las posibilidades pero no las saben usar, algo que ya hallamos en la época propiamente colonial:

They were barefooted and the tops of their white shirts floated outside their trousers. You may be laughing at shirt-tails outside trousers, but for the native this is the last word in elegance. I tried several times to get my boys to dress like you and me with the shirt tucked inside the trousers, but they said that that is for whites and continued to do it their own way (Bouteillier 1903, citado en Martin 1994: 407).

In his native dress, whether Moslem or pagan, the African is a dignified and picturesque figure: in the costume of Europe, as seen in the streets of Sierra Leone, he is a mere travesty of the white man, and unless dressed with considerable care and judgment becomes converted from an African into a nigger. Unfortunately it is almost impossible to bring the natives of districts in which Europeans are numerous to see this. Perceiving the general racial superiority of the white men, they consider it wise to imitate them, and, like many imitators, while letting essentials pass unnoticed they fix upon and closely copy mere externals (Freeman 1898: 380).

No resulta asimismo difícil encontrar esta misma idea en la narrativa actual:

No tarda en descubrir [...] a un hombrecillo [negro] con un antiguo traje de tergal demasiado grande, raído sombrero de fieltro y un par de zapatos de charol, brillantes como cucarachas enormes. Su figura muestra un aspecto tan ridículo en una primera ojeada — sobre todo bajo el sol implacable de la estación seca, que no parece posible tomarle en serio... (López Hidalgo 2007: 106-107).

Este conjunto de estrategias, que no hacen sino insistir en la inferioridad cultural de los africanos, no resultan visibles solo en los usos de la indumentaria, sino que tienen una especial relevancia en las modificacio-

${ }^{11} \mathrm{Y}$ al mismo tiempo los africanos critican con mucha frecuencia el desaliño de los europeos cuando están en África, algo que yo mismo he podido comprobar (véase también al respecto Nyamnjoh y Page 2002: 617-619).

${ }^{12} \mathrm{Al}$ respecto ver, por ejemplo, Martin (1994) y Thomas (2003). 
nes corporales. El caso de las mutilaciones genitales es bien actual hoy día, ya que desde hace algunas décadas ha ocupado y preocupado a Occidente, especialmente dentro del contexto ideacional de los denominados derechos humanos universales. La actual controversia es, no obstante, imposible de entender sin tener en cuenta los presupuestos de la colonialidad y, al margen del hecho de que constituyan un tipo de tradiciones que desde el punto de vista del individuo más valdría erradicar, los aspectos negativos de estas controversias son bien sencillos de entender. Siguiendo los clásicos esquemas de la colonialidad, esta manera de enfocar la problemática de las mutilaciones genitales en Occidente devalúa tanto a las personas como a las sociedades a las que pertenecen ${ }^{13}$. Las siguientes opiniones que podemos encontrar en foros de internet son bien explícitas al respecto:

[...] estamos viviendo en el siglo XXI; sin embargo en los países de casi toda África y algunos de la península arábica aun se vive con costumbres tan crueles y brutales como la ablación que consiste en la extirpación del clítoris de las mujeres para según sus hechores para evitar la "prostitución y el placer femenino", tan aberrante costumbre se practica en el "civilizado Egipto" y en 30 países más [...]. [...] Estamos viviendo la era del avance de la tecnología, donde cada vez es más fácil vivir y disfrutar de la vida, con menos horas de trabajo y más tiempo para el ocio; sin embargo esto es solo un espejismo si llegamos a las cálidas arenas africanas, donde la mujer es un ser de segunda clase; donde el hombre puede tener una o 20 mujeres y con casi todas engendra hijos, pero ninguna de estas mujeres pueden disfrutar del placer sexual, simplemente no tienen orgasmo y si así fuese es un delito dentro de la religión musulmana, y esto poco interesa al hombre o marido, ya que las mujeres solo son instrumentos para el placer masculino. La ablación como una inhumana costumbre es otra forma de violencia contra la mujer, que en nada se diferencia a las otras maneras de malos tratos, agresiones sexuales, golpazos, ataques armados con ventaja y alevosía contra las adoradas mujeres; realmente hasta ahora estoy sorprendido que en el mundo actual haya prácticas tan cavernarias como la extirpación del clítoris femenino que repugnan la conciencia humana ${ }^{14}$.

Y este es uno de los comentarios que en el mismo foro provocó el texto anterior:

Gonzalo te felicito con tu información sobre criminal costumbre de esos negros salvajes del África que extirpan el clítores [sic] a las mujeres, y todavía con dolor,

\footnotetext{
13 Véase, por ejemplo, Lane y Rubinstein (1996: 38).

${ }^{14}$ Subrayados en el original. Guillermo Gonzalo Sánchez Achutegui, URL : http:// piuraenlambayeque.blogspot.com/2008/12/la-ablacinuna-aberrante-extirpacin.html [fecha de consulta, abril de 2011]. Se trata de un blog personal editado desde el Perú y sin orientación política explícita que aborda temas muy diversos.
} 
que bestias negros, por eso pienso que los negros son tan salvajes como los monos que viven con ellos ${ }^{15}$.

Una cosa es pensar que existen prácticas culturales que sería mejor no realizar, y otra muy diferente continuar utilizando unos esquemas propios de los tiempos heroicos de la colonización, desde claras perspectivas etnocéntricas. Si hablamos de costumbres bárbaras estamos hablando obviamente de sus portadores también como bárbaros. Hablamos de colonialidad porque partimos de la base de que muchos de los presupuestos que se fueron generando durante el colonialismo continúan hoy día siendo vigentes. Y por lo que se refiere a la presentación social del cuerpo, uno de los casos donde esto se manifiesta de forma más clara es en la percepción que se tiene en Occidente sobre las mutilaciones genitales femeninas. Esta percepción se caracteriza por los siguientes rasgos:

\section{Desconocimiento}

En Occidente existe un gran desconocimiento por lo que se refiere a las prácticas de mutilación genital femenina. Esta es precisamente una de las críticas que la keniana Wairimu Ngaruiya Njambi hace al posicionamiento contrario a estas prácticas por parte del feminismo occidental (Njambi 2004: 293. Véase también Boddy 2007). En Occidente, cuando de forma genérica se habla de "ablación" se piensa en las formas más graves de este tipo de mutilaciones, como la infibulación o circuncisión faraónica, cuando de hecho según la tradición de la que se trate, las prácticas son muy diferenciadas y pueden consistir desde esta modalidad más severa hasta las que solamente implican una pequeña incisión efectuada en la corona del clítoris ${ }^{16}$.

\section{INTERPRETACIÓN REDUCCIONISTA}

Este desconocimiento implica interpretaciones sumamente reduccionistas ya que se entienden estas prácticas sobre todo como un método para controlar la sexualidad de las mujeres y como una muestra más de opresión masculina. Es cierto que esto puede ser así en algunas tradiciones (especialmente en el caso de la infibulación), pero no se debe generalizar en absolu-

15 URL : http://piuraenlambayeque.blogspot.com/2008/12/la-ablacinuna-aberranteextirpacin.html. Acceso, abril de 2011.

${ }^{16}$ Se estima que la infibulación constituye un $15 \%$ del total de este tipo de prácticas (Cf. Boddy 2007: 48). 
to ya que desde el punto de vista cuantitativo no es ni mucho menos la razón más importante. Se efectúan también por higiene, por estética, por creencias diversas y sobre todo por condicionantes sociales, dado que según el worldview de estas sociedades una mujer solo puede ser vista como tal - con todo lo que esto implica socialmente- si se somete a este rito de iniciación. Así, por ejemplo, Fuambai Ahmadu, antropóloga de Sierra Leona a quien también se le practicó la escisión, para su contexto cultural en concreto excluye radicalmente la relación de la mutilación genital con la opresión patriarcal y le reconoce un valor empoderador para la mujer a causa de las implicaciones de los rituales con los que va asociada (Ahmadu 2000).

En contra de la interpretación reduccionista está también el hecho de que en muchas sociedades africanas, a pesar de la práctica de la mutilación genital, se otorga la debida importancia al placer femenino en el coito y se aprenden técnicas para propiciarlo (Undie y Benaya 2006: 3; Njambi 2004: 296). Tenemos que recordar, además, que la amputación del clítoris, a pesar de la agresión física que presupone, no implica forzosa y automáticamente la anorgasmia, entre otras razones porque su eliminación puede conllevar un mayor desarrollo de otras zonas erógenas ${ }^{17}$.

\section{NATURALizaCión DE La idea OCCIDENTAL DE CUERPO}

Desde la órbita africana se critican con razón los estereotipos colonialistas del feminismo occidental por su etnocentrismo, apreciable en el hecho de presentar una imagen universalizada del cuerpo femenino que descansa en asunciones particularizadas de lo que se considera "normal" y "natural" ${ }^{18}$. Estamos hablando de otras cosmovisiones, no solo sobre la plasmación de la identidad masculina/femenina en determinadas características anatómicas, sino

${ }^{17}$ Véase al respecto, Lightfoot-Klein (1989); Obermeyer (1999: 95-96); Bonaparte (1953: 191-208); Gruenbaum (1996: 462). Tiene que hacer pensar también — sin que esto sirva de atenuante para este tipo de agresiones físicas- el hecho de que en las modernas operaciones de reconstitución genital de mujeres mutiladas, en un $80 \%$ de los casos el clítoris recupera su sensibilidad. Sobre la experiencia del orgasmo en mujeres circuncidadas véase muy especialmente Ahmadu (2007).

${ }^{18}$ Una primera asunción universalizada es que los cuerpos humanos son completos y el sexo es dado por nacimiento. Una segunda, que el clítoris representa un aspecto integral de la feminidad y tiene un papel central en la sexualidad de la mujer. Y una tercera, por extensión, considera la patriarcalidad como culpable; es decir las mujeres se ven como ciegas y bien dispuestas a aceptar la mutilación porque son víctimas de la dominación masculina en los ámbitos político, social y económico (Ahmadu 2000: 284). Véase también, Njambi (2004: 282). 
también sobre cómo se entiende la misma sexualidad y el placer. Según Fuambai Ahmadu, son precisamente las asunciones occidentales en relación al cuerpo de las mujeres más que los discutibles efectos sobre la salud de las operaciones genitales en mujeres africanas, lo que justifica las aversiones de los contrarios a estos tipos de prácticas (Ahmadu 2000: 284).

\section{DesconteXtualización}

Las mutilaciones genitales femeninas se encuentran en relación de complementariedad estructural con la circuncisión masculina. Con muy pocas excepciones, siempre que en una sociedad dada se practican las mutilaciones genitales femeninas se efectúan también las masculinas (Martí 2009: 231), aspecto que nos remite a significaciones de estos tipos de rituales que van más allá de las interpretaciones reduccionistas antes mencionadas. Y ya que aludimos a la circuncisión, también es digno de cuestionarse el hecho de que, a pesar de la condena que en Occidente se hace a las mutilaciones femeninas, este no es el caso para las masculinas, en las que se elimina la protección natural de una de las partes más sensibles del cuerpo. Es cierto que las consecuencias en el organismo son habitualmente más graves en el caso femenino que en el masculino, pero no podemos pasar por alto que una razón importante para la mayor indulgencia de nuestra opinión pública en relación a la circuncisión masculina se debe a que estamos mucho más familiarizados con ella y que constituye una práctica corriente en países como los EE. UU donde —además de los casos concretos de población judía o musulmana - se circuncida a un gran porcentaje de hombres también "por tradición" (Gollaher 2002: 253).

Desde un punto de vista objetivo y pensando en el individuo, no hay duda alguna de que prácticas como las mutilaciones genitales - masculinas o femeninas - son cuestionables. Pero pretender combatirlas con visiones etnocéntricas y alocrónicas herederas de las narrativas coloniales y cayendo además en la criminalización de los portadores de estas tradiciones, como a menudo sucede en Occidente, no es la mejor manera de conseguir su erradicación. El estado de opinión existente entre nosotros en contra de estas prácticas genera la movilización de asociaciones e individuos con finalidades solidarias y filantrópicas. El problema, y de esto evidentemente no somos siempre conscientes, es que más allá de estas loables finalidades, esta movilización, con toda la visibilidad mediática que la acompaña, desempeña la función de ayudar a mantener el paradigma intrínseco a la colonialidad que se basa en la creencia de la existencia de culturas superiores e inferiores. 
Otra consecuencia que podemos considerar también negativa, dado que estamos hablando de una serie de prácticas que implican agresiones físicas a los cuerpos, es que en determinados contextos culturales se generan procesos de resemantización de este tipo de prácticas, planteándose reivindicaciones de las mutilaciones genitales femeninas por razones identitarias. Estas reivindicaciones se han de ver como respuesta lógica ante la oposición occidental que es fácilmente percibida como imperialismo cultural (Gruenbaum 1995: 14-15). De hecho, ya mucho antes de que Occidente empezara a preocuparse de manera generalizada por las mutilaciones genitales femeninas, Jomo Kenyatta, político keniano formado en antropología en Londres con Bronislaw Malinowski, en contra de las prohibiciones de las autoridades coloniales, defendía la práctica de las mutilaciones genitales, dado que constituían una seña de identidad cultural de los kikuyu (Kenyatta 1971). Y en el año 2003 surgió un movimiento de cariz revivalista en Kenia, denominado Mungiki, que entiende la circuncisión femenina como uno de sus valores (Njambi 2004: 298). Así mismo, Michelle Johnson señala el valor identitario que las mutilaciones genitales femeninas han llegado a tener hoy día entre la población mandinga de Guinea Bissau (Johnson 2000). En palabras de Chantal Zabus: "While some women fight to remain 'whole women' with intact genitalia, others fight for the right to become 'whole' through excision" (Zabus 2008: 63).

Dado que todo lo que tiene que ver con la presentación social del cuerpo está estrechamente relacionado con la cuestión de las identidades, no nos debe extrañar que en África se haga bandera de ello cuando se trata de reafirmar la propia identidad frente a Occidente. Y si esto sucede con prácticas tan estigmatizadas como las de las mutilaciones genitales, es todavía más habitual con cuestiones de indumentaria. Bien sonada fue, por ejemplo, la prohibición, en la década de 1970, de Mobutu Sese Seko, presidente de Zaire - hoy República Democrática del Congo- de que los hombres de Kinshasa, la capital del país, llevasen el traje con americana y corbata al estilo occidental, imponiendo el abacost (es decir à bas le costume), para así señalizar la superación del pasado colonial. Son muchos los ejemplos que podríamos añadir al respecto, como el rechazo en los años 50 del siglo pasado al uso del sombrero en Accra como acto de afirmación identitaria y de oposición al poder colonial y a la anglofilia de algunas élites urbanas (Allman 2004: 1); el rechazo al traje victoriano entre los movimientos nacionalistas yoruba de finales del siglo XX (Byfield 2004: 31); el movimiento de los Creoles en Sierra Leone a finales de 1880, que propugnaba la creación de una indumentaria propia que los distinguiese, tanto de los europeos, como de las comunidades indígenas (Byfield 2004: 34); la indumentaria autóctona entendida como resistencia ante los poderes coloniales 
ingleses e italianos en Somalia a partir ya de finales del siglo XIX (Akou 2004: 50); aparte de otras disposiciones que, ligadas a la idea de decencia pública, prohíben también el uso de determinadas piezas de indumentaria considerada occidental, como por ejemplo los pantalones para las mujeres, casos que hoy día se producen en el $\operatorname{Sudan}^{19}$ o Swazilandia ${ }^{20}$.

\section{CODA: LA BURLA DE LA CULTURA}

En la Enciclopedia Espasa (1925 vol. 27: 285), en el artículo que hace referencia a Guinea Ecuatorial se dice, entre otras cosas, que los tatuajes propios de la población de la costa continental se continúan practicando con la excepción de aquellos casos en que los individuos "descienden de una familia en que el padre ha experimentado la influencia de la civilización". Este artículo fue redactado en las primeras décadas del siglo $\mathrm{XX}^{21}$. Curiosamente, no obstante, modificaciones corporales muy similares a aquellas que se consideraban propias de los incivilizados las podemos ver hoy día en nuestra civilización entre los practicantes del body art más actual. La costumbre de llevar la camisa por fuera de los pantalones, y que podía servir para ridiculizar a los negros, tal como hemos visto anteriormente en un ejemplo procedente de una publicación de 1903, es hoy también algo propio de nuestra modernidad. Actualmente, en muchas playas africanas es posible ver mujeres europeas tomando el sol con los senos descubiertos ante los ojos atónitos de las mujeres autóctonas que se los cubren pudorosamente, después de que sus madres o abuelas fuesen bien aleccionadas en cuestiones de decencia por los misioneros cristianos. Y si tenemos que hablar de desnudez, a la fuerza hay que mencionar el auge del movimiento nudista europeo o incluso el progresismo de la normativa de la ciudad de Barcelona de 2004, que permitía ir completamente desnudo por las calles de la ciudad (Martí 2010b) 22 .

\footnotetext{
${ }^{19}$ Amnesty International, "Amnesty International calls on Sudan to repeal law penalizing women for wearing trousers", 4.9.2009, URL: http://www.amnesty.org/en/news-andupdates/news/amnesty-international-sudan-repeal-law-penalizing-women-wearing-trousers20090904. Fecha de consulta, septiembre de 2011.

20 "Multa a las mujeres con pantalones", La Vanguardia, 7.5.2003, p. 13 (vivir).

${ }^{21}$ Hoy día solo conservan estos tatuajes muy pocas personas y todas ellas ya ancianas. El tatuaje tradicional fang dejó de practicarse a lo largo de la primera mitad del siglo XX.

${ }^{22}$ Esta normativa fue derogada en mayo del 2011. Si se derogó no fue sin embargo por las molestias que pudieran ocasionar los pocos activistas nuditistas que se paseaban sin ropa alguna por la ciudad, sino por la mala imagen que según algunos daban de Barcelona los turistas que en época estival deambulaban por el centro de la ciudad
} 
En ocasiones, no se duda en recurrir a esta misma presentación social del cuerpo que los esfuerzos de los administradores coloniales consiguieron erradicar en buena medida, cuando de lo que se trata es de hacer negocio con el cuerpo del nativo en el contexto del denominado realismo turístico. En un artículo de Bruner y Kirshenblatt-Gimblett sobre las performances turísticas que se llevaban a cabo hasta hace algunos años en una hacienda en Kenia, administrada por descendientes de colonos británicos, en la que se ofrecía a los turistas escenas de la vida de los maasai, aparecen muy claras las normas que se imponían a los autóctonos que se mostraban ante los turistas: nada de mostrar relojes digitales, T-shirts o calcetines deportivos, radios ni walkman. Se cuidaba de que se decoraran el cuerpo y el cabello a la manera tradicional, y la indumentaria con la que se cubrían el cuerpo debía ser también de aquel color rojo con que generalmente más se asocia a los maasai (Bruner y Kirshenblatt-Gimblett 1994: 457). Los propietarios de aquella hacienda exhortaban a sus trabajadores a ser maasai, "ya que si no lo parecían suficientemente, los tour-operadores no les aportarían turistas" (Ibid.: 459) ${ }^{23}$.

Curiosamente, aquello que a los ojos de los europeos aparecía en épocas pasadas como claras muestras de inferioridad cultural de los africanos resulta que hoy es celebrado por determinados sectores de nuestra población como signo de modernidad. Esto no hace sino recordarnos el carácter en principio arbitrario que tienen muchos rasgos culturales, más allá de toda veleidad evolucionista; una arbitrariedad, sin embargo, que aparentemente se desvanece cuando les otorgamos significaciones concretas en el tiempo y en el espacio. Y aquí podríamos hablar de la "burla de la cultura": lo que antes a ellos los hacía salvajes, a nosotros ahora nos hace modernos. En el África francófona, en época colonial, se utilizaba el término évolué, en un principio para designar aquellos africanos que habían pasado a formar parte de la modernidad, pero entrando en descrédito, el término ha acabado por asumir unas connotaciones más bien burlonas. No hace falta decir, que nosotros, occidentales, en relación con los africanos siempre querríamos ser

con atuendo playero. Habría resultado paradójico que se hubiera prohibido por ley el semidesnudo de los turistas en sus paseos por la Rambla y se siguiera permitiendo el desnudo integral de los nuditistas.

${ }_{23} \mathrm{Al}$ respecto resulta también interesante el caso que nos describe Britta Duelke (2002). Un Aboriginal Arts Centre del norte de Australia en el que a finales de los años 80 se conminaba a los hombres y mujeres de la comunidad donde se hallaba el centro a que se mostraran ante los potenciales turistas según el estilo de vida tradicional, lo que entre otras cosas implicaba exhibirse en desnudez y la renuncia a usar comodidades modernas en el espacio público. 
los évolués, y esto tiene obviamente mucho que ver con la construcción de la colonialidad. Y en el contexto de esta colonialidad, el establecimiento de identidades es crucial para construir un orden social jerarquizado, y este resulta imprescindible para dotar de legitimidad a determinados patrones en las relaciones de intercambio entre individuos marcados con una $\mathrm{u}$ otra etiqueta, unas relaciones que en el marco del colonialismo devienen fácilmente sinónimo de explotación. En suma, tal y como hemos podido ir viendo a lo largo de este artículo, todo lo que incumbe a la presentación social del cuerpo no es en absoluto ajeno a estos procesos de colonialidad.

\section{BIBLIOGRAFÍA CITADA}

Aguayo. 1943. "Exposiciones escolares". La Guinea española 1163: 260.

Akou, H. M. 2004. "Nationalism without a Nation: Understanding the Dress of Somali Women in Minnesota", en J. Allman (ed.), Fashioning Africa: Power and the Politics of Dress: 50-63. Bloomington: Indiana University Press.

Ahmadu, F. 2000. "Rites and Wrongs: an Insider/Outsider Reflects on Power and Excision", en B. S. Duncan y Y. Hernlund (eds.), Female "circumcision" in Africa: culture, controversy, and change: 283-312. Boulder: Lynne Rienner Publishers.

Ahmadu, F. 2007. "Ain't I a Woman Too?': Challenging Myths of Sexual Dysfunction in Circumcised Women", en Y. Hernlund y B. Shell-Duncan (eds.), Transcultural Bodies: Female Genital Cutting in Global Context: 278-310. New Brunswick-Londres: Rutgers University Press.

Allman, J. 2004a. "Introduction to Fashioning Africa: Power and the Politics of Dress", en J. Allman (ed.), Fashioning Africa: Power and the Politics of Dress: 1-12. Bloomington: Indiana University Press.

Allman, J. 2004b. "Let Your Fashion Be in Line with Our Ghanaian Costume': Nation, Gender, and the Politics of Cloth-ing in Nkrumah's Ghana", en J. Allman (ed.), Fashioning Africa: Power and the Politics of Dress: 144-165. Bloomington: Indiana University Press.

Amnesty International 2009. Amnesty International calls on Sudan to repeal law penalizing women for wearing trousers, 4-9-2009. http://www.amnesty.org/en/ news-and-updates/ news/amnesty-international-sudan-repeal-law-penalizing-women-wearing-trousers20090904. Acceso, septiembre de 2011.

Ardener, E. W. 1954. "Some Ibo Attitudes to Skin Pigmentation". Man 54: 71-73.

Aymemí, A. 1942. Los bubis en Fernando Póo. Madrid: Galo Sáez.

Aymemí, A. 1943. "De la familia bubi". La Guinea española 1149: 46-47.

Bakale-Yosuf, B. 2011. "Nudity and morality: legislating women's bodies and dress in Nigeria", en S. Tamale (ed.), African Sexualities: A Reader: 116-129. Cape Town: Pambazuka.

Beltrán y Rózpide, R. s.f. La Guinea española. Barcelona: Manuel Soler Editor. Manuales Soler 17.

Blay, Y. A. 2011. "Skin Bleaching and Global White Supremacy: By Way of Introduction". The Journal of Pan African Studies 4 (4): 4-46. 
Boddy, J. 2007. "Gender Crusades: The Female Circumcision Controversy in Cultural Perspective", en Y. Hernlund y B. Shell-Duncan (eds.), Transcultural Bodies: Female Genital Cutting in Global Context: 46-66. New Brunswick-Londres: Rutgers University Press.

Bonaparte, M. 1953. Female Sexuality. Nueva York: International Universities Press.

Bonelli Rubio, J. M. 1949. "Diferencia del concepto económico en la colonización de Fernando Póo y Guinea Continental". Archivos del Instituto de Estudios Africanos 3: 7.

Bouteillier, G. 1903. Douze mois sous l'equateur. Toulouse: Imprimerie Adolphe Trinchant.

Briault, M. 1943. Les sauvages d'Afrique. París: Payot.

Bruner y B. Kirshenblatt-Gimblett. 1994. "Maasai on the Lawn: Tourist Realism in East Africa”. Cultural Anthropology 9 (4): 435-470.

Byfield, J. 2004. "Dress and Politics in Post-World War II Abekuta (Western Nigeria)", en J. Allman (ed.), Fashioning Africa: Power and the Politics of Dress: 31-49. Bloomington: Indiana University Press.

Butler, J. 1988. "Performative Acts and Gender Constitution: An Essay in Phenomenology and Feminist Theory". Theatre Journal 40 (4): 519-531.

Castro-Gómez, S. 2005. La poscolonialidad explicada a los niños. Popayán: Editorial Universidad del Cauca.

Droz, Y. 2009. "La morale de l'interdiction de la clitoridectomie en pays kikuyu". Anthropologie et Sociétés 33 (3): 118-138.

Duelke, B. 2002. "Nakedfella oder Varianten der Nacktheit", en K. Gernig (ed.), Nacktheit. Ästhetische Inszenierungen im Kulturvergleich: 167-184. Köln: Böhlau.

Dy'Ikengue, I. 2004. Costumbres bengas y de los pueblos vecinos (editado por P. Rabat Makambo). Madrid: Sial (edición original: 1892. Customs of the Benga and Neighboring Tribes. Nueva York: H. E. Simmons).

Enciclopedia Universal Ilustrada. 1925. Bilbao-Madrid-Barcelona: Espasa-Calpe.

Fanon, F. 1973. Piel Negra, Máscaras Blancas. Buenos Aires: Abraxas.

Ferguson, J. G. 2002. "Of Mimicry and Membership: Africans and the 'New World Society". Cultural Anthropology 17 (4): 551-569.

Fontán y Lobé, J. 1943. "La etnología y la política indígena". Conferencia http:// www.asodegue.org/hcdf1d.430626.htm. Acceso, abril de 2011.

Fortes, M. 1945. The Dynamics of Clanship among the Tallensi. Londres: Oxford University Press.

Freeman, R. A. 1898. Travels and life in Ashanti and Jaman. Londres: A. Constable.

Gollaher, D. 2002. Das verlezte Geschlecht. Die Geschichte der Beschneidung. Berlín: Aufbau-Verlag.

Goody, E. y J. Goody. 1996. "The Naked and the Clothed", en I. Wilks; J. O Hunwick y N. E. Lawler (eds.), The cloth of many colored silks: papers on history and society, Ghanaian and Islamic in honor of Ivor Wilks: 67-89. Evanston: Northwestern University Press.

Gruenbaum, E. 1995. "Women's Rights and Cultural Self-Determination in the Female Genital Mutilation Controversy". Anthropology Newsletter May: 14-15.

Gruenbaum, E. 1996. "The Cultural Debate Over Female Circumcision: The Sudanese Are Sorting This One Out for Themselves". Medical Anthropology Quarterly 10 (4): 455-475.

Hamond, W. 1640. A Paradox. Prooving, That the Inhabitants of the Isle called Madagascar, Or St. Laurence, (In Temporall things) are the happiest People in the World. Londres: Printed for Nathaniell Butter. 
Hay, M. J. 2004. "Changes in Clothing and Struggles over Identity in Colonial Western Kenya", en J. Allman (ed.), Fashioning Africa: Power and the Politics of Dress: 144165. Bloomington: Indiana University Press.

Heinz, A. 1998. "Colonial Perspectives in the Construction of the Psychotic Patient as Primitive Man". Critique of Anthropology 18: 421-444.

Hendrickson, H. 1996. Clothing and difference: embodied identities in colonial and postcolonial Africa. Durham-Londres: Duke University Press.

Hunter, M. L. 2007. "The persistent problem of colorism: Skin tone, status, and inequality". Sociology Compass 1: 237-254.

Johnson, M. "Becoming a Muslim, Becoming a Person: Female 'Circumcision', Religious Identity, and Personhood in Guinea-Bissau", en B. S. Duncan y Y. Hernlund (eds.), Female "circumcision" in Africa: culture, controversy, and change: 215-234. Boulder: Lynne Rienner Publishers.

Kapuscinski, R. 2004. Ébano. Barcelona: Ediciones Folio.

Kenyatta, J. 1971. Facing Mount Kenya. The tribal Life of the Gikuyu. Nairobi: Heinemann Educational Books.

Lander, R. y J. Lander. 1844. Journal of an Expedition to Explore the Course and Termination of the Niger. Nueva York: Harper \& Brothers, 2 vols.

Lane, S. D y R. A. Rubinstein. 1996. "Judging the other: Responding to traditional female genital surgeries". Hastings Centre Report 26 (3): 31-40.

Le Breton, D. 2002. La sociología del cuerpo. Buenos Aires: Nueva Visión.

Lightfoot-Klein, H. 1989. "The Sexual and Marital Adjustment of Genitally Circumcised and Infibulated Females in the Sudan". Journal of Sex Research 26 (3): 375-392.

López Hidalgo, J. A. 2007. La casa de la palabra, Santa Cruz de Tenerife: Idea.

López Medel, T. 1990. Colonización de América: informes y testimonios 1549-1572. Madrid: Consejo Superior de Investigaciones Científicas.

Magubane, B. 1971. "A critical look at indices used in the study of social change in colonial Africa". Current Anthropology 12: 419-45.

Manchester Chamber of Commerce. 1884. Address of Mr. H. Stanley. Manchester: A. Ireland. Martí, J. 2009. "Modificaciones corporales en la tradición africana", en Y. Aixelà, L. Mallart y J. Martí (eds.), Introducción a los Estudios Africanos: 221-238. Vic: CEIBA.

Martí, J. 2010a. "La presentación social del cuerpo: Apuntes teóricos y propuestas de análisis", en J. Martí y Y. Aixelà (eds.), Desvelando el Cuerpo. Perspectivas desde las ciencias sociales y humanas: 107-122. Madrid: CSIC.

Martí, J. 2010b. "Passejar-se nu per Barcelona, avui". Quaderns de l'Institut Català d'Antropologia 26: 159-180.

Martin, P. M. 1994. "Contesting Clothes in Colonial Brazzaville". The Journal of African History 35 (3): 401-426.

Martín del Molino, A. 1994. La ciudad de Clarence. Madrid: Centro Cultural Hispano Guineano.

Mauss, M. 1971. Sociología y antropologia. Madrid: Tecnos.

McClintock, A. 1995. Imperial Leather: Race, Gender and Sexuality in the Colonial Contest. Nueva York: Routledge.

Mediavilla, H. 2008. "La moda como orgullo: Los sapeurs". La Vanguardia (Magazine) 7-12-2008.

Medina-Doménech, R. 2009. "Scientific Technologies of National Identity as Colonial Legacies: Extracting the Spanish Nation from Equatorial Guinea". Social Studies of Science 39 (1): 81-112. 
Mirzeler, M. y C. Young. 2000. "Pastoral politics in the northeast periphery in Uganda: AK-47 as change agent". The Journal of Modern African Studies 38 (3): 407-429.

Miyanji de Souza, M. 2008. "The concept of skin bleaching in Africa and its devastating health implications". Clinics in Dermatology 26 (1): 27-29.

"Multa a las mujeres con pantalones". 2003. La Vanguardia, 7-5-2003: 13.

Nandy, A. 1983. The Intimate Enemy. Loss and Recovery of Self under Colonialism. Delhi: Oxford University Press.

Natsoulas, T. 1998. "The Politicization of the Ban on Female Circumcision and the Rise of the Independent School Movement in Kenya: The KCA, the Missions and Government, 1929-1932". Journal of Asian and African Studies 33: 137-158.

Nerín, G. 1998. Guinea Ecuatorial, historia en blanco y negro: hombres blancos y mujeres negras en Guinea Ecuatorial (1843-1968). Barcelona: Península.

Njambi, W. N. 2004. "Dualisms and female bodies in representations of African female circumcision: A feminist critique». Feminist Theory 5: 281-303.

Nyamnjoh, F. B. y B. Page. 2002. "Whiteman Kontri and the Enduring Allure of Modernity among Cameroonian Youth". African Affairs 101 (405): 607-634.

Obermeyer, C. M. 1999. "Female Genital Surgeries: The Known, the Unknown and the Unknowable». Medical Anthropology Quarterly 13: 79-106.

Peña, C. T. de la. 2006. "'Bleaching the Ethiopian': Desegregating race and technology through early X-ray experiments". Technology and Culture 47: 27-55.

Restrepo, E. 2007. "Antropología y colonialidad", en S. Castro-Gómez y R. Grosfoguel (eds.), El giro decolonial. Reflexiones para una diversidad epistémica más allá del capitalismo global: 289-304. Bogotá: Siglo del Hombre Editores.

Reischer, E. y K. S. Koo. 2004. "The Body Beautiful: Symbolism and Agency in the Social World". Annual Review of Anthropology 33: 297-317.

Rich, J. 2005. "Civilized attire: Refashioning tastes and Social Status in the Gabon Estuary, c. 1870-1914". Cultural and Social History 2: 189-213.

Ruether, K. 2002. "Heated Debates over Crinolines: European Clothing on NineteenthCentury Lutheran Mission Stations in the Transvaal. Journal of Southern African Studies 28 (2): 359-378.

Ruiaz. 1944. "Galería de Coloniales. El Excmo. P. Nicolás González". La Guinea Española 1166: 20-22.

Ruiaz. 1944. "Mis impresiones". La Guinea Española 1170: 91-92.

Sabater, J. y J. O. Sabater. 1992. Els tatuatges dels Fang de l'Àfrica Occidental. Barcelona: Ajuntament de Barcelona.

Sánchez Molina, E. R. 2002. "Homo Infantilis. Asimilación y segregación en la política colonial española en Guinea Ecuatorial". Revista de Dialectología y Tradiciones Populares 57 (2): 105-120.

Scheper-Hughes, N. y M. M. Lock. 1987. "The Mindful Body: A Prolegomenon to Future Work in Medical Anthropology". Medical Anthropology Quarterly, New Series 1 (1): 6-41.

Schneider, L. 2006. “The Maasai's New Clothes: A Developmentalist Modernity and Its Exclusions". Africa Today 53 (1): 101-129.

Simon, H. A. 1960. The New Science of Management Decision. Nueva York: Harper and Row.

Thomas, D. 2003. “Fashion Matters: 'La Sape' and Vestimentary Codes in Transnational Contexts and Urban Diasporas". MLN 118 (4): 947-973. 
Thomas, L. M. 2011. "Bodily Conceptions and Biomedical Opposition to Skin Lighteners in Apartheid South Africa". Comunicación presentada en la 4th European Conference on African Studies. Uppsala: ECAS4-The Nordic Africa Institute.

Undie, C. y K. Benaya. 2006. "The state of knowledge on sexuality in sub-saharan Africa: a synthesis of literature". Jenda: a Journal of Culture and African Women Studies 8: $1-14$.

Walker, A. 1983. In Search of Our Mother's Gardens. Nueva York: Harcourt Brace Jovanovich.

Wright, L. B. 1943. "The noble savage of Madagascar in 1640". Journal of the History of the Ideas 4 (1): 112-118.

Zabus, C. 2008. "From 'Cutting Without Ritual' to 'Ritual Without Cutting': Voicing and Remembering the Excised Body in African Texts and Contexts", en M. F. Borch, E. R. Knudsen y M. Leer (eds.), Bodies and Voices: The Force-Field of Representation and Discourse in Colonial and Postcolonial Studies: 45-68. Amsterdam-Nueva York: Rodopi.

Fecha de recepción: 20 de noviembre de 2011

Fecha de aceptación: 29 de febrero de 2012 